\title{
What is meant by a "controlled" ventricular rate in atrial fibrillation?
}

John M Rawles

\begin{abstract}
Reduction of a rapid ventricular rate in atrial fibrillation results in a longer diastolic filling period and a higher left ventricular stroke volume but this is offset by reduced contractility and fewer beats per minute; the net effect on cardiac output is uncertain. Sequences of stroke distances were measured by Doppler ultrasound in 60 resting patients with atrial fibrillation to determine the relation between ventricular rate and linear cardiac output. The slope of the cardiac output/ventricular rate relation was positive in all 20 patients with a ventricular rate $<90$ beats per minute and negative in $16(40 \%)$ of 40 patients with a ventricular rate $>90$ beats per minute. In atrial fibrillation the ventricular rate can be regarded as "controlled" when the cardiac output/ ventricular rate slope is positive and "uncontrolled" when the slope is negative-that is when reduction of ventricular rate would lead to increased cardiac output. As so defined, ventricular rate at rest was controlled in every patient when the ventricular rate was $<90$, controlled in $44(73 \%)$ patients when the ventricular rate was $90-140$ beats per minute, and uncontrolled in every case when it was $>140$ beats per minute.

Achieving a target ventricular rate of 90 beats per minute in patients with atrial fibrillation at rest would result in control with the least compromise of cardiac output.
\end{abstract}

Reduction of a rapid ventricular rate in atrial fibrillation increases the diastolic filling period and left ventricular stroke volume, ${ }^{12}$ but this is offset by reduced contractility and fewer beats per minute. The net effect on cardiac output is uncertain. A standard textbook recommends maintaining the ventricular rate at $50-90$ beats per minute at rest and $90-115$ beats per minute during exercise, ${ }^{3}$ with the implicit intention of maximising cardiac output. None the less, the ventricular rate at which cardiac output in atrial fibrillation is maximal is not known nor is the extent of individual variation.

The objectives of this study in patients with atrial fibrillation were to develop a method of predicting the effect of a change of ventricular rate on cardiac output, to determine the ventricular rate for maximal cardiac output, and to find the extent of the variation between individuals. The concept of "control" of the ventricular rate in atrial fibrillation was reexamined in the light of these findings. The intention here is not to apply the method to individual patients but to see what general conclusions can be drawn about the relation between ventricular rate and cardiac output in atrial fibrillation.

In atrial fibrillation there is spontaneous beat to beat variation of ventricular rate around the mean value: analysis of the effect of these changes on stroke output makes it possible to estimate the effect of a change in mean ventricular rate on cardiac output. Once the relation between previous RR intervals and stroke output is obtained it can be transformed to give the relation between ventricular rate and cardiac output.

Most of the beat to beat variation of stroke output can be explained in terms of the two previous $R R$ intervals used in a quadratic polynomial equation. ${ }^{4}$ Adjustment of each patient's unique equation enables the ventricular rate at which cardiac output is maximal to be estimated together with the slope of the cardiac output/heart rate relation. Only if the slope is negative will a reduction of ventricular rate lead to an increase of cardiac output.

\section{Patients and methods}

I studied 60 patients (24 women and 36 men, mean age 68, mean ventricular rate 103 beats per minute) with chronic atrial fibrillation, who were derived from a consecutive series of 70 . In 10 recordings were unreadable in parts. Atrial fibrillation was idiopathic in 26 and in the remainder it was caused by mitral valve disease (13), ischaemic heart disease and/or hypertension (19), thyrotoxicosis (one), and pulmonary embolism (one). Twenty six patients were treated with digoxin, four with amiodarone, and one with a $\beta$ blocker. Twenty nine patients were not treated with antiarrhythmic agents.

In each patient aortic blood flow was measured by continuous wave ultrasound directed at the arch of the aorta from a transducer held in the suprasternal notch. ${ }^{5}$ The Doppler shift frequencies were subjected to spectral analysis and displayed in real time on a paper trace on which a single lead of the electrocardiogram was co-recorded at a paper speed of $50 \mathrm{~mm} / \mathrm{s}$. The stroke distance (systolic velocity integral) of each beat was measured by triangulation or planimetry and the sequence of $R R$ intervals and stroke distances was recorded on disc. The mean number of beats studied was 215 (range 100-500). For each patient multiple regression analysis of stroke distance against 
the two previous $R R$ intervals was carried out according to the equation:

$$
\begin{gathered}
S d=a+b(R R-1)+c(R R-1-750)^{2}+ \\
d(R R-2)+e(R R-2-750)^{2}
\end{gathered}
$$

where $\mathrm{Sd}$ is stroke distance, $\mathrm{RR}-1$ the previous RR interval, $R R-2$ the pre-previous $R R$ interval, and a to e are coefficients. The derivation of this mathematical model has been fully described. ${ }^{4}$

Rearrangement of this equation gives the relation between heart rate and minute distance (minute distance $=$ stroke distance $\times$ heart rate), from which the predicted heart rate at which minute distance would be expected to be maximal was obtained, together with the slope of the minute distance/heart rate curve at the point of mean heart rate. The slope was expressed as minute distance (as a percentage of the age predicted normal value)/(beats per minute).

The result of the computer analysis was available within a few minutes of taking the ultrasound recording at the patient's bedside.

\section{Results}

\section{MEAN STROKE AND MINUTE DISTANCE}

For the whole group of 60 patients with atrial fibrillation mean stroke distance was $64 \%$ and mean minute distance $85 \%$ of the age adjusted normal values for sinus rhythm ${ }^{5}-$ a highly significant reduction in each case $(p<0.001)$. Minute distance was reduced less than stroke distance because ventricular rate was above normal. The mean value of the multiple correlation coefficient, relating stroke distance to $R R$ intervals in each individual, was $\mathbf{0 . 8 2}$ (range 0.68-0.94).

RELATION BETWEEN SLOPE OF MINUTE DISTANCE/ HEART RATE CURVE AND VENTRICULAR RATE

Figure 1 shows the relation between the slope of the minute distance/heart rate curve and ventricular rate. For lower heart rates the slope was positive and for higher rates it was negative; the regression line intercepted the line of slope zero, corresponding to maximal cardiac output, at a heart rate of 122 per minute. On average, reduction of heart rate below this rate will reduce minute distance; above this rate minute distance will be increased, but there is wide individual varia-

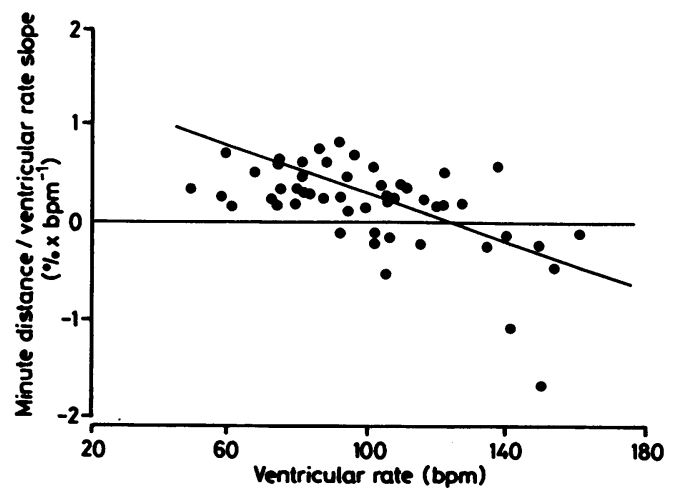

Figure 1 Plot of the slope of the minute distancel ventricular rate curve and mean ventricular rate in patients with atrial fibrillation. The regression line crosses the line of slope zero at a ventricular rate of 122 .

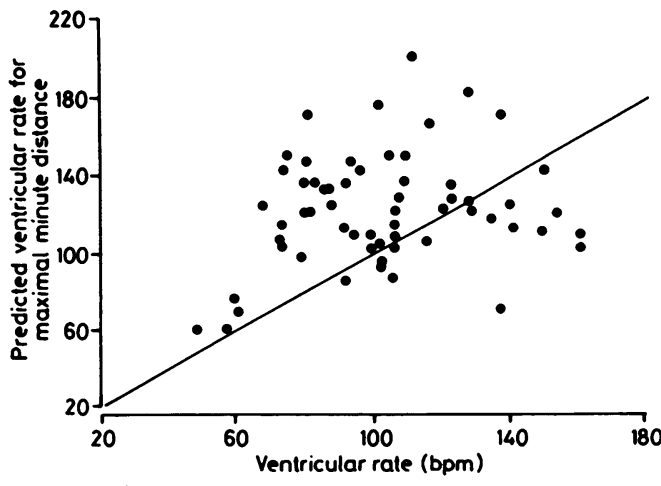

Figure 2 Plot of mean ventricular rate and predicted ventricular rate for maximal minute distance. The line of identity is shown.

tion. The average slope for the seven patients with ventricular rates of more than 140 per minute is -0.65 , indicating an increase of minute distance of $6.5 \%$ of the age-predicted normal value for a reduction of ventricular rate of 10 beats per minute.

PREDICTED VENTRICULAR RATE FOR MAXIMAL MINUTE DISTANCE

The group mean (SD) ventricular rate was 103 $(26 \cdot 6)$ beats per minute (range $49-161$ beats per minute). The mean predicted ventricular rate for maximal minute distance, derived from the multiple regression equations, was $122(28.8)$ (range $60-200$ beats) per minute. Figure 2 shows a plot of ventricular rate and predicted ventricular rate for maximal minute distance. In $44\left(73^{\circ}{ }_{0}\right)$ cases the predicted ventricular rate for maximal minute distance was more than the measured ventricular rate (above the line of identity in fig 2) but in $16(27 \%)$ it was lower (below the line of identity in fig 2). All 16 patients with a ventricular rate above that predicted for maximal minute distance had a ventricular rate over 90 beats per minute. In none of the 20 patients with a ventricular rate $<90$ did the ventricular rate exceed the predicted ventricular rate for maximal minute distance. In all seven patients with a ventricular rate of $\geqslant 140$ the ventricular rate exceeded that predicted for maximal cardiac output, and the slope of the minute distance/ventricular rate curve was therefore negative.

Figure 3 shows the cumulative total of patients with various ventricular rates and the number of patients whose ventricular rates exceeded that predicted for maximal outputthat is those whose minute distance/ventricular rate slopes were positive (“controlled").

EFFECT OF IRREGULARITY ITSELF ON MINUTE DISTANCE

The multiple regression equation was obtained by analysis of the beat to beat changes of stroke distance resulting from a sequence of $R R$ intervals of varying duration. If the value of the mean RR interval for the sequence is substituted in the multiple regression equation then stroke distance, and hence minute distance, is calculated for the circumstances in which all RR intervals have an identical duration. The difference between this value and measured minute distance for the sequence is 


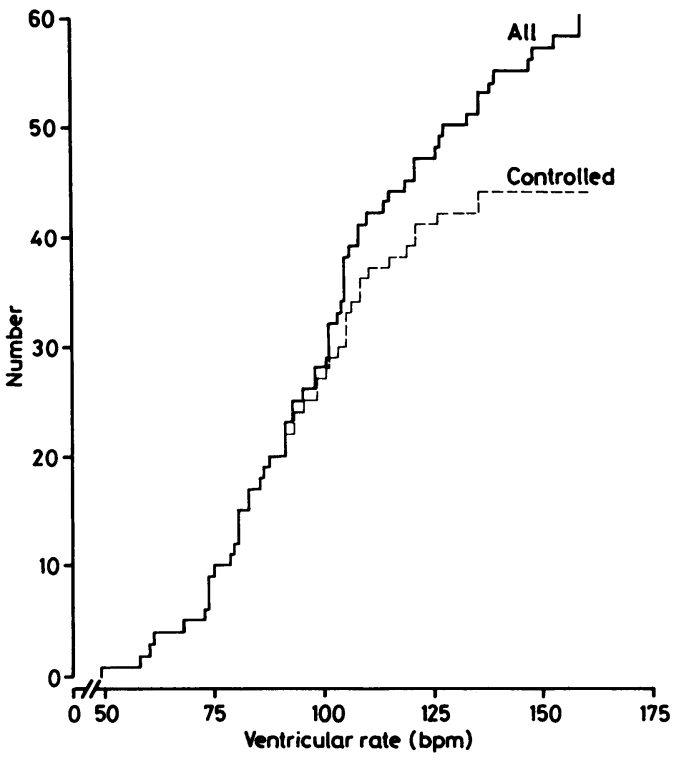

Figure 3 Cumulative totals of all patients and those with "controlled" atrial fibrillation at various ventricular rates.

an indication of the effect of irregularity itself on minute distance. For the whole group, measured minute distance (SD) was $96(3 \cdot 2 \%)$ of the calculated value, indicating that irregularity of ventricular rate results in an average fall of minute distance of $4 \%$.

There were no distinctive differences in the multiple correlation coefficients, minute distance, or predicted ventricular rate for maximal cardiac output between different aetiological groups or treatments.

\section{Discussion}

I measured linear cardiac output noninvasively using Doppler ultrasound. The basic measurement is the systolic velocity integral of aortic blood flow or stroke distance; the product of stroke distance and ventricular rate is minute distance. Stroke and minute distance are linear analogues of stroke volume and volumetric cardiac output respectively. ${ }^{6}$

Beat to beat changes of stroke distance can be described mathematically in terms of the two previous RR intervals in this group of patients with atrial fibrillation. ${ }^{4}$ In every patient there was a positive relation between stroke distance and the previous RR interval (RR-1) which was thought to reflect predominantly the diastolic filling of the left ventricle. In all but one patient there was a negative relation with the preprevious $R R$ interval (RR-2) that was attributed to augmentation of contractility by the force-frequency effect of the shorter RR intervals. Change in the mean duration of $R R$ interval will affect stroke distance not only through diastolic filling of the left ventricle but also by affecting contractility.

Because the mathematical model is based on the physiological principles underlying left ventricular function it can be applied to any case of atrial fibrillation, whatever the ventricular rate, however long the arrhythmia has been present, and however it is caused.
EFFECT OF VENTRICULAR RATE ON CARDIAC OUTPUT

Analysis of the beat to beat changes in stroke distance in patients with atrial fibrillation is used to predict the ventricular rate for maximal cardiac output. This is done by summation of the independent effects of the two previous RR intervals on strake distance to derive the effect of alteration of the mean RR interval on stroke distance, and hence to predict how mean ventricular rate would influence linear cardiac output. The most reliable section of the minute distance/ventricular rate curve is in the region of the mean ventricular rate where the slope of the curve (that is, whether it is negative or positive) determines the answer to the clinically important question, "If the ventricular rate is reduced will cardiac output rise or fall?"

The slope of the minute distance/ventricular rate curve was always positive for ventricular rates below 90 beats per minute, and as a corollary the predicted ventricular rate for maximum minute distance was always greater than the actual ventricular rate. Conversely, the slope was always negative for ventricular rates above 140 beats per minute, when the predicted ventricular rate for maximum minute distance was lower than the measured ventricular rate. The regression line of slope against ventricular rate passed through zero at a ventricular rate of 122 beats per minute, indicating that on average this was the ventricular rate at which minute distance was maximal (fig 1). The mean predicted value of ventricular rate for maximum cardiac output was also 122 beats per minute. In atrial fibrillation with various causes the average predicted ventricular rate for maximal cardiac output at rest is therefore about 120 beats per minute, with a wide individual variation ranging from 60 to 200 beats per minute. There were no distinctive differences in the shape, position, or fit of the curve relating ventricular rate to linear cardiac output between different aetiological or treatment groups.

All of 16 patients whose ventricular rate exceeded that predicted for maximal cardiac output had a resting ventricular rate greater than 90 beats per minute. Patients with a ventricular rate in excess of that predicted for maximal cardiac output accounted for $40 \%$ of those with a ventricular rate over $90,58 \%$ of patients with a ventricular rate over $110,80 \%$ of those with a ventricular rate over 130 , and all those with a ventricular rate over 140 beats per minute.

\section{EFFECT OF IRREGULARITY ITSELF ON CARDIAC} OUTPUT

If the mean $R R$ interval for each patient is substituted into the multiple regression equation it is possible to calculate the value of minute distance when the mean ventricular rate is identical with that measured, but the rhythm is regular with all $R R$ intervals being of equal duration. The difference between minute distance calculated in this way and measured directly is an indication of the effect on minute distance of irregularity of rate itself. On average, measured minute distance was $4^{\circ} \%$ less 
than calculated minute distance, suggesting that irregularity of heart rate by itself is not an important cause of reduced cardiac output in atrial fibrillation. The effect of irregularity on cardiac output is determined by the symmetry and shape of the minute distance/heart rate graph and on the frequency distribution of beats with stroke distance above and below average.

\section{COMPARISON OF ATRIAL FIBRILLATION AND SINUS} RHYTHM

Atrial fibrillation reduces stroke volume by a loss of atrial contraction, but an increased ventricular rate partially compensates for this and the reduction of cardiac output caused by the arrhythmia is estimated to be $20-30 \%{ }^{78}$ In the present series, stroke and minute distance were reduced by $36 \%$ and $15 \%$ respectively, and the mean ventricular rate at 103 beats per minute was $48 \%$ higher than the normal values for sinus rhythm predicted by age. ${ }^{5}$ The tachycardia of atrial fibrillation is therefore usually a beneficial phenomenon partially counteracting an even greater fall of cardiac output that would otherwise occur, except in those patients in whom the actual ventricular rate exceeds the rate for maximal output.

In our patients with atrial fibrillation, who had a mean ventricular rate of 103 , the mean minute distance was $85 \%$ of the age-adjusted normal value for patients in sinus rhythm, but if ventricular rate had been reduced to the normal resting value of 70 the estimated minute distance would have been only about $70 \%$ of normal.

WHAT IS MEANT BY "CONTROL" OF VENTRICULAR RATE?

When it is applied to the ventricular rate in atrial fibrillation the term "controlled" is generally used loosely to mean "not excessive". Few define precisely what is meant by a controlled rate but Bigger specified 50-90 beats per minute, ${ }^{3}$ while Storstein suggested an even narrower range of $60-80$ beats per minute; both referred to patients at rest. The mid point of both these ranges is 70 , the accepted normal rate in sinus rhythm. However, in sinus rhythm the contribution made to stroke volume by atrial contraction is estimated to be $20-50 \%$, depending on left ventricular compliance and other aspects of left ventricular function. ${ }^{1011} \mathrm{In}$ atrial fibrillation, therefore, the ventricular rate needs to be $20-50 \%$ higher than in sinus rhythm to maintain a normal cardiac output. The recommendation of a target ventricular rate of 70 in atrial fibrillation seems to disregard completely the haemodynamic consequences of the arrhythmia.

The prediction of the effect of ventricular rate on cardiac output described in this paper is derived from analysis of recordings made from patients with atrial fibrillation at rest. The results should not be extrapolated to exercise, in which - as well as a change of heart ratethere may be alterations of posture, venous return, blood pressure, cardiac contractility, and autonomic tone. The heart rate for maximal cardiac output during exercise therefore differs from that at rest. In sinus rhythm rates of up to 180 beats per minute are achieved during exercise, and it seems likely that rates at least as great as this may be required in atrial fibrillation to maximise cardiac output.

Some workers use the term "control" to mean avoidance of excessive ventricular rates during exercise. Thus David et al reported a mean ventricular rate of 139 beats per minute during exercise in patients with therapeutic concentrations of serum digoxin; they wrote that "digitalis alone is ineffective in controlling the heart rate during exercise in many patients with chronic atrial fibrillation". ${ }^{12}$ Beasley et al compared ventricular rates in patients with atrial fibrillation and serum concentrations of digoxin $>1.6 \mathrm{ng} / \mathrm{l}$ with those in controls in sinus rhythm. ${ }^{13}$ At rest and at three levels of exercise ventricular rates were $72,105,118,130$ in sinus rhythm and $79,135,162$, and 184 in atrial fibrillation. They considered that in atrial fibrillation the heart rate during exercise was inappropriately high and pointed out that it is not predictable from a knowledge of the resting value. The ventricular rate could therefore be "controlled" at rest but "uncontrolled" during exercise.

Channer et al conducted a randomised crossover trial of additional digoxin or verapamil in 14 patients with atrial fibrillation who were already taking digoxin. ${ }^{14}$ Although the additional treatment resulted in lower maximal heart rates, the distance walked in 6 minutes was unchanged. Lewis et al gave single doses of digoxin, verapamil, and diltiazem, alone and in combination, to six patients with chronic atrial fibrillation. ${ }^{15}$ Immediately after exercise lower heart rates were seen with the combination of digoxin and calcium antagonists than with digoxin alone or with placebo. Reduction of the ventricular rate during exercise was associated with a small increase of stroke volume but the benefits of this were offset by a rate related reduction in cardiac output. The highest mean cardiac output was after digoxin alone when the mean ventricular rate was 159 beats per minute. Mean walking distances were not significantly different after any of the treatments.

These data indicate that exercise tolerance is not increased by reduction of ventricular rate. During exercise and at rest a higher ventricular rate is required in atrial fibrillation than in sinus rhythm to maintain cardiac output in the face of the loss of the atrial contribution to stroke output.

A NEW CONCEPT OF CONTROL OF THE VENTRICULAR RATE IN ATRIAL FIBRILLATION

In atrial fibrillation it may be necessary to reduce a rapid ventricular rate for the greater comfort of the patient, or, if mitral stenosis is present, to reduce left atrial pressure and relieve pulmonary oedema. However, in the management of atrial fibrillation the effect that a reduction of rate will have on cardiac output should be considered: ventricular rate should not be reduced arbitrarily irrespective of its effect on cardiac output or exercise tolerance.

I have described a method that for patients at 
rest provides an answer to the question posed earlier: "If the ventricular rate in atrial fibrillation is reduced will cardiac output rise or fall?" In principle the method could be applied to patients during exercise, but with existing equipment the quality of the recordings would be inadequate for accurate interpretation. Even at rest continuous records of sufficiently good quality could not be obtained in some individuals. The reliability of individual predictions will be the subject of further reports, but from this study on 60 resting patients with atrial fibrillation some general observations can be made.

A "controlled" ventricular rate in atrial fibrillation can be regarded as a rate below that predicted for maximal cardiac output-that is when the slope of the cardiac output/ventricular rate curve is positive and a reduction of rate would lead to a reduction of output. When ventricular rate is "controlled" the circulatory reflexes may adjust the rate according to circulatory needs. Conversely, an "uncontrolled" ventricular rate is above that predicted for maximal cardiac output and reduction of rate would lead to increased output; reflex activity would tend to exacerbate the tachycardia. As so defined, ventricular rate was always controlled at $<90$ beats per minute, always uncontrolled at $>140$ beats per minute, and uncontrolled in $27^{\circ}$ of patients with ventricular rates between 90 and 140 .

In atrial fibrillation, a target ventricular rate of 90 beats per minute at rest would be within the range where it can be adjusted by the cardioregulatory reflexes, and cardiac output would be least compromised. During exercise the heart rate that results in maximal cardiac output is likely to be greater than the target rate of $90-115$ that has been recommended. ${ }^{3}$

I thank Professor M J R Healy for many helpful comments on the derivation of the mathematical model and on an earlier draft of the paper.

I thank the Grampian Health Board for a research grant.

1 Wood P. Diseases of the heart and circulation. 2nd ed. London: Eyre and Spottiswoode, 1958:254.

2 Friedberg CK. Diseases of the heart. 3rd ed. Philadelphia: WB Saunders, 1966:537.

3 Bigger JT. Management of arrhythmias. In: Braunwald E, ed. Heart disease. Philadelphia: WB Saunders, 1980:731.

4 Rawles JM. A mathematical model of left ventricular function in atrial fibrillation. Int $J$ Biomed Comput 1988;23:57-68.

5 Haites NE, McLennan FM, Mowat DHR, Rawles JM Assessment of cardiac output by the Doppler ultrasound technique alone. Br Heart J 1985;53:123-9.

6 Haites NE, McLennan FM, Mowat DHR, Rawles JM. How far is the cardiac output? Lancet 1984;ii: 1025-7.

7 Ferrer MI, Harvey RM, Cathcart RT, Cournand A Richards DW. Haemodynamic studies in rheumatic heart disease. Circulation 1952;6:668-710.

8 Wade G, Werko L, Eliasch H, Gidlund A, Lagerlof $H$. The haemodynamic basis of the symptoms and signs in mitral valvular disease. $Q J$ Med 1952;21:361-83.

9 Storstein L. Role of digitalis in ventricular rate control in atrial fibrillation. In: Kulburtus $\mathrm{HE}$, Olsson $\mathrm{SB}$, Schlepper M, eds. Atrial fibrillation. Molndal: AB Hassle, 1984:288.

10 Rahimtoola SH, Ehsani A, Sinno MZ, Loeb HS, Rosen KM, Gunnar RM. Left atrial transport function in myocardial infarction. Am $J$ Med 1975;59:686-94.

11 DeMaria AN, Miller RR, Amsterdam EA, Markson W, Mason DT. Mitral valve early diastolic closing velocity: relation to sequential diastolic flow and ventricular compliance. Am J Cardiol 1976;37:693-700.

12 David D, Di Segni E, Klein HO, Kaplinsky E. Inefficiency of digitalis in the control of heart rate in patients with chronic atrial fibrillation: beneficial effects of an added beta adrenergic blocking agent. Am J Cardiol 1979;44:1378-82.

13 Beasley R, Smith DA, McHaffie DJ. Exercise heart rate at different serum digoxin concentrations in patients with atrial fibrillation. Br Med J 1985;290:9-11.

14 Channer KS, Papouchado M, James MA, Pitcher DW, Rees JR. Towards improved control of atrial fibrillation. Eur Heart $J$ 1987;8:141-7.

15 Lewis R, Irvine N, McDevitt DG. Relationships between heart rate, exercise tolerance and cardiac output in atrial fibrillation: the effects of treatment with digoxin, verapamil and diltiazem. Eur Heart $J$ 1988;9:777-81. 